Article

\title{
Some Chaos Notions on Dendrites
}

\author{
Asmaa Fadel $\mathbb{B}$ and Syahida Che Dzul-Kifli * \\ Center of Modelling and Data Sciences, Faculty of Science and Technology, Universiti Kebangsaan Malaysia, \\ 43600 Bangi, Malaysia; asma-1011@hotmail.com \\ * Correspondence: syahida@ukm.edu.my; Tel.: +603-89213425
}

Received: 15 September 2019; Accepted: 8 October 2019; Published: 17 October 2019

check for updates

\begin{abstract}
Transitivity is a key element in a chaotic dynamical system. In this paper, we present some relations between transitivity, stronger and alternative notions of it on compact and dendrite spaces. The relation between Auslander and Yorke chaos and Devaney chaos on dendrites is also discussed. Moreover, we prove that Devaney chaos implies strong dense periodicity on dendrites while the converse is not true.
\end{abstract}

Keywords: dendrite; transitive; locally everywhere onto; mixing; weakly mixing; blending; accessible endpoint; Devaney chaos; Auslander and Yorke chaos; strong dense periodicity.

\section{Introduction}

It is worth mentioning some important notions for our work. By the pair $(X, f)$, we mean a dynamical system where $X$ is a compact metric space and $f$ is a continuous function (map) on $X$. The symbols $\mathbb{N}$ and $\mathbb{R}$ represent the set of natural and real numbers, respectively. The orbit of a point $x \in X$, denoted by $\operatorname{Orb}(x)$, is the set $\left\{f^{m}(x): m \in \mathbb{N} \cup\{0\}\right\}$ and a point that has a dense orbit is called a transitive point. In addition, the boundary (resp. cardinality) of a set $A \subseteq X$ will be denoted by $\partial A$ (resp. $|A|$ ).

During the last few decades, chaos has become one of the most crucial topics in the dynamical system. And therefore, many concepts of chaos were presented. However, there is no global agreement on the most accurate chaos concept to describe chaotic behavior. It turns out that Devaney chaos [1] is the most frequently used definition of chaos. In this concept, the Devaney chaotic dynamical system $(X, f)$ poses three conditions:

1. transitive (for any non-empty open sets $U$ and $V, \exists m \in \mathbb{N}$ s.t $f^{m}(U) \cap V \neq \varnothing$ ),

2. contains a dense set of periodic points,

3. sensitive dependence on initial conditions (SDIC) $(\exists \alpha>0$, s.t $\forall x \in X$ and $\forall \beta>0, \exists y \in X$ and $\exists m \in \mathbb{N}$ s.t $d(x, y)<\beta$ but $\left.d\left(f^{m}(x), f^{m}(y)\right) \geq \alpha\right)$.

It was proven that $S D I C$ is redundant on Devaney chaos whenever $X$ is an infinite compact space [2]. Moreover, Vellekoop and Berglund [3] proved that Devaney chaos coincides to transitive on the interval. Another remarkable chaos notion is Auslander and Yorke chaos [4]. The dynamical system $(X, f)$ is called Auslander and Yorke chaos if it is transitive and SDIC.

Since transitivity is the main ingredient in a chaotic dynamical system, many stronger or alternative concepts to it were constructed. Some examples of definitions are stronger than transitivity: locally everywhere onto (for every non-empty open set $U \subseteq X, \exists m \in \mathbb{N}$ s.t $f^{m}(U)=X$ ), mixing (for any non-empty open sets $U, V \subseteq X, \exists M \in \mathbb{N}$ s.t $\left.f^{m}(U) \cap V \neq \varnothing, \forall m \geq M\right)$, weakly mixing $(f \times f$ is transitive) 
and totally transitive ( $f^{m}$ is transitive $\forall m \in \mathbb{N}$ ). The following implication is obvious in any compact metric space:

Locally everywhere onto $\Rightarrow$ Mixing $\Rightarrow$ Weakly mixing $\Rightarrow$ Totally transitive $\Rightarrow$ Transitive.

In [5], the definitions of strongly and weakly blending were defined as alternative concepts to transitivity, since they are more intuitive. We said that the dynamical system $(X, f)$ is weakly blending if, for any non-empty open sets $U$ and $V, \exists m \in \mathbb{N}$ s.t $f^{m}(U) \cap f^{m}(V) \neq \varnothing$ and it is strongly blending when $f^{m}(U) \cap f^{m}(V)$ contains a non-empty open set. On the interval, neither strongly blending nor weakly blending implies transitivity and the converse is also not true $[5,6]$.

A stronger notion of dense periodicity, strong dense periodicity, was introduced by Dzul-Kifli [7]. We say that the system $(X, f)$ has strong dense periodicity, if $\forall m \in \mathbb{N}$, the set

$$
P_{m}(f)=\{x: x \text { is periodic point of period greater than or equal to } m\}
$$

is dense. The works in [6-11] discussed the relation between strong dense periodicity and other notions of chaos on intervals, circles and shift of finite space.

Studying which concepts imply the others and finding the minimal sufficient conditions for the relations to be held is one of the most important ideas in dynamical systems. It was proven that a totally transitive map on a compact space that has a dense set of periodic points implies weakly mixing [12]. According to [5], a strongly blending map on a subset $A \subseteq \mathbb{R}^{n}$, whose set of periodic points is dense, implies transitivity. Moreover, on compact space, locally everywhere onto implies strongly blending [10], while mixing is sufficient to imply weakly blending [6]. On the other hand, Banks and Trotta [13] proved that weakly mixing implies strongly blending on the graph.

By a continuum, we mean a non-empty compact connected metric space. A metric space $(X, d)$ is said to be a Peano space if, for any point $x \in X$ and every neighborhood $N$ of $x$, there exists an open connected set $U \subseteq N$ containing $x$. A Peano continuum space that contains no simple closed curve is called a dendrite $(D)$. Refs. $[14,15]$ are two important works talking about dendrites. Let $\alpha$ be a cardinal number and let $x \in D$. We said that the point $x$ is of order $\alpha$, denoted by $\operatorname{Ord}(x, D)=\alpha$, if, for any open set $V$ containing $x$ and any cardinal number $\beta<\alpha$, there is an open set $U$ containing $x$ contained in $V$ such that $\beta<|\partial U| \leq \alpha$. In dendrites, the order of $x$ is equal to the number of components of $D \backslash\{x\}$ whenever one of them is finite. The point $x \in D$ whose $\operatorname{Ord}(x, D)=1$ is called an endpoint where $x$ is a cut point if $\operatorname{Ord}(x, D) \geq 2$ and branch point if $\operatorname{Ord}(x, D) \geq 3$.

The two most familiar examples of dendrites are the closed interval and tree (a dendrite with a finite set of endpoints) which are also special cases of graphs. By an arc, we mean any space homeomorphic to the closed interval $[0,1]$. We denote the arc that has two endpoints $x$ and $y$ as $x y$. Every dendrite $D$ admits a taxicab metric, a metric where for every arc $x y$ in $D$ and every point $z \in x y, d(x, y)=d(x, z)+d(z, y)$. A set $E$ of topological space $X$ is called a free interval if it is open in $X$ and homeomorphic to an open interval in $\mathbb{R}$. A subset $H$ of a connected space $X$ is a disconnecting interval if it is a free interval and $\forall x \in H$, $X \backslash\{x\}$ has only two components [16]. A dendrite whose set of branch points is not dense (equivalently, the set of endpoints is not dense) is an example of a space containing a disconnecting interval.

In the study of a one-dimensional dynamical system, it is natural to ask whether we can extend any existing results on the interval to more general spaces. In literature, some results on trees, circles, graphs and dendrites have been discussed as an analogue to the results on intervals. The interest in the study of chaotic behaviour on dendrites has been increasing for a few years. For examples, researches focus on shadowing [17], distributional chaos [18], induced map [19], and relations between some chaotic notions [20-23]. It turns out that most of the results on the interval do not necessarily hold true on dendrites. However, this is not the case for dendrites with special properties (which relate to its endpoints). For example, on the interval mixing and weakly mixing are equivalent and transitivity implies dense 
periodic points. However, there is a counterexample on a dendrite, which proved that these results do not hold true on dendrites [22,23]. Surprisingly, these results are true on dendrites whose set of branch points is not dense [16,21]. For dendrites whose set of endpoints is countable, transitivity $=$ Devaney chaos [20].

Let $f$ be a map on the interval $[a, b]$. The endpoint $a$ (resp. $b$ ) is said to be accessible if $\exists x \notin\{a, b\}$ and $m \in \mathbb{N}$ s. $\mathrm{t} f^{m}(x)=a$ (resp. $f^{m}(x)=b$ ). Blokh [24] constructed a relation between mixing and locally everywhere onto an interval map. He proved that a mixing map on $[a, b]$ is locally everywhere onto if and only if $a$ and $b$ are accessible. In [25], Ruette pointed out that this result can be extended to a graph.

Motivated by these studies, in this paper, we discuss the generalization of Blokh's result on a dendrite map. We prove that locally everywhere onto implies accessibility of endpoints if the set of branch points of dendrites is not dense and provide a counterexample to show that it is not true in general. For the converse, we give a prove to show that, if $f$ is a tree mixing map and the set of endpoints is accessible, then $f$ is locally everywhere onto. As far as we are concerned, our proof is a new proof for this result on intervals. Furthermore, we generalize the previous result to any dendrite with a taxicab metric. Our main results are about some chaotic concepts on compact and dendrite spaces. On compact space, we prove that the existence of a transitive point, whose every open set containing it covers the space after some iterations, is sufficient for the space to be locally everywhere onto. We also found that transitivity and strongly blending are sufficient for a compact map to be mixing. We also show that weakly mixing implies weakly blending on any dendrite and weakly mixing implies strongly blending for dendrites that do not have a dense set of branch points. In addition, the relation between Devaney chaos and Auslander and Yorke chaos on dendrites is also discussed. It is well known that Devaney chaos implies Auslander and Yorke chaos for any compact space. We show that the converse is not true on dendrites or compact space. Finally, we prove that Devaney chaos implies strong dense periodicity on dendrites, but the converse is not true. These results are contained in the next section.

This paper is arranged as follows. Section 2 contains our results while the conclusion is presented in Section 3.

\section{Results}

Our contributions in this paper are some implication relations of chaos notions on dendrites. In some cases, the implications only hold true for special cases of dendrites: dendrites whose set of branch points is not dense, interval, and tree. Some examples are also provided to show untrue implications. However, we also prove some implications on more general space: compact space.

Relations we are going to discuss are between locally everywhere onto, mixing and accessibility of endpoints.

Definition 1. Let $f: D \rightarrow D$ be a dendrite map and $e$ be an endpoint of $D$. Then, $e$ is said to be accessible if $\exists x \in D \backslash \operatorname{End}(D)$ and $m \in \mathbb{N}$ such that $f^{m}(x)=e$.

Theorem 1. Let $f: D \rightarrow D$ be a map on a dendrite whose set of branch points is not dense. Then, locally everywhere onto implies the accessibility of each endpoint of $D$.

Proof. Let $D$ be a dendrite whose set of branch points is not dense. Then, the set of endpoints is also not dense. Thus, there exists a non-empty open set $U$ in $D$ such that $U$ does not contain any endpoint of $D$. Since $f$ is locally everywhere onto then $\forall x \in D, \exists a \in U$ and $m \in \mathbb{N}$ s.t $f^{m}(a)=x$. For each endpoint of $D$, such $a$ is a cut point. Therefore, each endpoint is accessible.

Now, we illustrate that the previous theorem is not valid in general. 
Proposition 1. Let $f: D \rightarrow D$ be a dendrite map. Then, locally everywhere onto does not imply that every endpoint of $D$ is accessible.

Proof. In [23], the authors constructed a locally everywhere onto map on a dendrite whose set of transitive points (points with dense orbit) is a subset from the set of endpoints. We claim that there exist some endpoints in that example which are not accessible. Assume that every endpoint is accessible. Let $e$ be a transitive endpoint, since $e$ is accessible then $\exists a \in D \backslash E n d(D)$ and $m \in \mathbb{N}$ such that $f^{m}(a)=e$. Since $e$ is a transitive point, so is $a$, which contradicts the fact that every transitive point is an endpoint.

Ruette [25] pointed out that the mixing graph map whose every endpoint is accessible implies locally everywhere onto. In the following part, we will construct a proof for this theorem on tree map. Our proof can also be used on an interval map.

Theorem 2. Let $f: T \rightarrow T$ be a mixing tree map. $f$ is locally everywhere onto if and only if every endpoint of $T$ is accessible.

Proof. First, suppose $T$ contains $n$ endpoints. Let

$$
\operatorname{End}(T)=\left\{e_{i}: i=1,2, \ldots, n\right\}
$$

be the set of endpoints of $T$ and

$$
A=\left\{a_{i}: i=1,2, \ldots, n\right\}
$$

be a set of cut points, where $\forall i=1,2, \ldots, n, \exists n_{i} \in \mathbb{N}$ s.t $n_{i}$ is the least natural number that satisfies $f^{n_{i}}\left(a_{i}\right)=e_{i}$. Since $T$ is normal, for each $e_{i}: i=1,2, \ldots, n$, we can find an open set $U_{i}$ containing $e_{i}$ and disjoint with the set $A \cup \operatorname{End}(T) \backslash\left\{e_{i}\right\}$. Now, choose $W$ to be any non-empty open set, $f$ is mixing so, for every open set $U_{i}, \exists m_{i} \in \mathbb{N}$ s.t

$$
f^{M_{i}}(W) \cap U_{i} \neq \varnothing, \quad \forall M_{i} \geq m_{i} .
$$

Next, let $k=\max \left\{m_{i}: i=1,2, . ., n\right\}$. Then, $\forall l \geq k, f^{l}(W)$ is a connected set containing the set A. Finally, if $t=\max \left\{n_{i}: i=1,2, . ., n\right\}$, then $f^{k+t}(W)=T$. Conversely, the same way as the proof in Theorem 1.

Theorem 3 ([22]). Let $f$ be a map on a continuum space $X$. $f$ is mixing if and only if $\forall \epsilon>0$ and for all non-empty open set $U, \exists M \in \mathbb{N}$ such that

$$
d_{H}\left(f^{m}(U), X\right)<\epsilon, \quad \forall m \geq M,
$$

where $d_{H}\left(f^{m}(U), X\right)$ is the Hausdorff distance.

Theorem 4. Let $d$ be a taxicab metric on a dendrite $D$ and $f$ be a mixing map on $D$. If every endpoint of $D$ is accessible, then $f$ is locally everywhere onto.

Proof. Without loss of generality, let $U$ be any non-empty open connected set in $D$ with a taxicab metric, $A$ be a set defined as

$$
A=\left\{x \in D \backslash \operatorname{End}(D): f^{n}(x) \in \operatorname{End}(D) \text { for some } n \in \mathbb{N}\right\}
$$

and $\delta=\min \{d(e, A): e \in \operatorname{End}(D)\}$.

Choose $\epsilon=\delta / 2$. Then, since $f$ is mixing, $\exists M \in \mathbb{N}$ s.t

$$
d_{H}\left(f^{m}(U), X\right)<\epsilon, \forall m \geq M .
$$


Claim $A \subseteq f^{m}(U), \forall m \geq M$. Assuming not, $\exists a \in A$ and $a \notin f^{m}(U)$ for some $m \geq M$. Then, there exists an endpoint $e$ such that $e a \cap f^{m}(U)=\varnothing$. $e \notin f^{m}(U)$, therefore $\exists y \in f^{m}(U)$ s.t $d(e, y)<\epsilon$. Now, $e, a$ and $y$ are in the same arc (otherwise, $e a \cup a y \cup e y$ is a closed curve in $D$ ). Since $d$ is taxicab,

$$
d(e, y)=d(e, a)+d(a, y) .
$$

We know that

$$
d(e, y)<\epsilon \quad \text { where } \quad d(e, a)+d(a, y)>2 \epsilon+d(a, y) .
$$

This implies that

$$
2 \epsilon<d(e, y)<\epsilon,
$$

which is a contradiction. Therefore,

$$
A \subseteq f^{m}(U), \quad \forall m \geq M
$$

Thus,

$$
\exists n \in \mathbb{N} \quad \text { s.t } \quad f^{n}(U)=D .
$$

In the next part, we construct a theorem connecting transitivity and locally everywhere onto on a compact space.

Theorem 5. Let $(X, f)$ be a transitive dynamical system on a compact space and $x \in X$ be a transitive point. If, for each open set $V$ containing $x$, there exists $n \in \mathbb{N}$ such that $f^{n}(V)=X$, then $f$ is locally everywhere onto.

Proof. Let $U$ be any non-empty open set in $D$ and $x$ is a transitive point satisfying the condition in the theorem. Since the orbit of $x$ is dense, there exist $n \in \mathbb{N}$ such that $f^{n}(x) \in U$. Thus, $V=f^{-n}(U)$ is an open set containing $x$. Therefore, $\exists m \in \mathbb{N}$ such that

$$
X=f^{m}(V)=f^{m}\left(f^{-n}(U)\right) \subseteq f^{m-n}(U) .
$$

Thus, $f$ is locally everywhere onto.

The following theorem, which is related to weakly mixing, is due to Banks [26].

Theorem 6 ([26]). Let $f$ be a map on a space $X$. $f$ is weakly mixing if and only if, for any non-empty open sets $U$ and $V, \exists m \in \mathbb{N}$ s.t $f^{m}(V) \cap U \neq \varnothing$ and $f^{m}(V) \cap V \neq \varnothing$.

Next, there are relations among mixing, blending and transitivity.

Theorem 7. Let $f$ be a map on a compact metric space $X$. If $f$ is transitive and strongly blending, then $f$ is weakly mixing.

Proof. Let $U$ and $V$ be two non-empty open sets. $f$ is strongly blending, so there exists $k \in \mathbb{N}$ such that $f^{k}(U) \cap f^{k}(V) \supseteq M$, where $M$ is a non-empty open set. Now, $f$ is transitive and $M, V$ are two non-empty open sets; therefore, there exists $l \in \mathbb{N}$ such that $f^{l}(M) \cap V \neq \varnothing$. Since

$$
M \subseteq f^{k}(U) \cap f^{k}(V),
$$

we have

$$
f^{l}(M) \subseteq f^{l}\left(f^{k}(U) \cap f^{k}(V)\right) \subseteq f^{l+k}(U) \cap f^{l+k}(V)
$$


Hence,

$$
f^{l+k}(U) \cap f^{l+k}(V) \cap V \neq \varnothing .
$$

It follows that

$$
f^{l+k}(U) \cap V \neq \varnothing \quad \text { and } \quad f^{l+k}(V) \cap V \neq \varnothing .
$$

Thus, $f$ is weakly mixing.

Since weakly mixing and mixing are equivalent on dendrites whose branch points are not dense, we get the following theorem.

Theorem 8. Let $f$ be a transitive map on a dendrite whose set of branch points is not dense. Then, strongly blending implies mixing.

The following example will be important for our work.

Example 1. Let $h:[0,1] \rightarrow[0,1]$ be defined as

$$
h(x)= \begin{cases}2 x+2, & \text { if }-1 \leq x<\frac{-1}{2} \\ -2 x, & \text { if } \frac{-1}{2} \leq x<\frac{1}{2} \\ 2 x-2, & \text { if } \frac{1}{2} \leq x \leq 1\end{cases}
$$

Remark 1. Strongly blending is essential in Theorem 7 and can not be replaced by weakly blending. To illustrate this, let $h(x)$ be the map defined on Example 1. Crannell [5] investigated that $h$ is transitive weakly blending but not a strongly blending map. It follows that, since $h$ does not have a periodic point of odd period greater than $1, h$ is not weakly mixing. Therefore, transitive weakly blending compact map is not weakly mixing.

Now, two crucial definitions for our following work will be presented.

Definition 2. Let $A$ and $B$ be two non-empty connected disjoint sets in a dendrite. An arc $C$ is called a connected $\operatorname{arc}$ of $A$ and $B$ if $A \cup B \cup C$ is a connected set.

Definition 3. Let $A$ and $B$ be two non-empty connected disjoint sets in a dendrite. An arc $H$ is called the minimal connected arc of $A$ and $B$ if $H$ is a connected arc of $A$ and $B$ and, for any connected arc $C$ of $A$ and $B, H \subseteq C$.

We will now show that the minimal connected arc defined in Definition 3 is unique.

Lemma 1. Let $A, B$ be two non-empty connected disjoint sets in a dendrite $D$ and for any $x \in D, A \cup B \cup\{x\}$ is a disconnected set. Then, $A$ and $B$ has a minimal connected arc and it is unique.

Proof. Let $A$ and $B$ be two non-empty connected disjoint sets in a dendrite $D$. Define the set

$$
H=\left\{\cap J_{\alpha}: \alpha \in \Delta, J_{\alpha} \text { is a connected arc of } A \text { and } B\right\} .
$$

Claim, if $H \neq\{x\}$, where $x \in D$, then it is the minimal connected arc of $A$ and $B$.

Notation: Sometimes, we will write the connected arc $J_{\alpha}$ of $A$ and $B$ as $x y$, where $x, y \in D$.

First, we will show that $H \neq \varnothing$. To do this, we need to prove that every two connected arcs of $A$ and $B$ are not disjoint. Now, let $x y$ and $x_{1} y_{1}$ be two disjoint connecting arcs of $A$ and $B$. Then,

$$
[A \cup B \cup x y] \cup\left[A \cup B \cup x_{1} y_{1}\right]
$$


is a connected set. Thus, there exists two arcs $x x_{1}$ and $y y_{1}$ in it. Now,

$$
x y \cup x x_{1} \cup x_{1} y_{1} \cup y y_{1}
$$

is a closed curve, which contradicts the definition of dendrites.

Now, to prove that $H \neq \varnothing$. Assume the opposite i.e., $H=\varnothing$. Then, $\exists x \in a b$, where $a b$ is a connected arc of $A$ and $B, a \in A, b \in B$ and $x \notin A \cup B$. We can find another connected arc of $A$ and $B$ say $a_{1} b_{1}$, where $a_{1} \in A, b_{1} \in B$ and $x \notin a_{1} b_{1}$. Since any two arcs are not mutually disjoint, $\exists y \in a_{1} b_{1} \cap a b$. Three cases for the position of $y$ can be found.

Case 1: $y \in A$, in this case, we get the closed curve $y b \cup b b_{1} \cup y b_{1}$ in $A \cup B \cup a b \cup a_{1} b_{1}$, which is a contradiction.

Case 2: $y \in B$, in this case, we get the closed curve $y a \cup a a_{1} \cup y a_{1}$ in $A \cup B \cup a b \cup a_{1} b_{1}$, which is a contradiction.

Finally: if $y \notin A \cup B$, then either we have $y b \cup b b_{1} \cup y b_{1}$ or $y a \cup a a_{1} \cup y a_{1}$ as a closed curve in $A \cup B \cup a b \cup a_{1} b_{1}$, which is also a contradiction. Therefore, $H \neq \varnothing$.

Next, to prove that $H$ is connected, suppose that $H$ is a disconnected set. Then, $H=U \cup V$, where $U \cap V=\varnothing$. Let $u \in U$ and $v \in V$. Since $u$ and $v \in H$, then $u, v \in J_{\alpha}, \forall \alpha \in \Delta$, which is a connected set so there exists an arc $u v \subseteq J_{\alpha}, \forall \alpha \in \Delta$. It follows, since the arc $u v$ is unique in $D$, that $u v \subseteq H=U \cup V$, which contradicts the fact that $U$ and $V$ are disjoint and their union is disconnected. Hence, $H$ is connected. Clearly, $H$ is a closed set.

We proved that $H$ is a continuum set in $\mathrm{D}$ so it is a subdendrite since every subcontinuum of dendrite is a dendrite. Now, we need to show that $C$ is an arc. By the fact, if $Y$ is a subdendrite of $D$, then $|\operatorname{End}(Y)| \leq|\operatorname{End}(D)|$; we can say that, for all $\alpha,|\operatorname{End}(H)| \leq\left|\operatorname{End}\left(J_{\alpha}\right)\right|$. Therefore, $|\operatorname{End}(H)| \leq 2$, which implies that $H$ is an arc.

To prove that $H$ is connected arc of $A$ and $B$, we need to show that $L=A \cup B \cup H$ is a connected set:

$$
L=A \cup B \cup H=A \cup B \cup \bigcap_{\alpha \in \Delta} J_{\alpha}=\bigcap_{\alpha \in \Delta}\left(A \cup B \cup J_{\alpha}\right)=\bigcap_{\alpha \in \Delta} L_{\alpha} .
$$

For each $\alpha \in \Delta, L_{\alpha}$ is a connected set and any two sets are not disjoint. Thus, by the same strategy which was used to prove that $H$ is connected, we can prove that $L$ is also connected.

Finally, we will show the uniqueness of $H$. Suppose that $H$ and $I$ are two minimal connected arcs of $A$ and $B$. $H$ is minimal; then, $H \subseteq I$ and also $I$ is minimal, so $H \subseteq I$. Thus, $H=I$.

The next theorem that relates to weakly mixing is due to Furstenberg [27].

Theorem 9 ([27]). Let $f$ be a weakly mixing map on a space $X$; then,

$$
\forall m \in \mathbb{N},(\underbrace{X \times X \times \ldots \times X}_{m}, \underbrace{f \times f \times \ldots \times f}_{m})
$$

is transitive.

Next, we prove that weakly mixing implies weakly blending on dendrites.

Theorem 10. On dendrites, weakly mixing implies weakly blending.

Proof. Let $U$ and $V$ be two non-empty open sets and, without loss of generality, assume they are disjoint connected sets (If $U \cap V \neq \varnothing$, then $f^{n}(U) \cap f^{n}(V) \neq \varnothing, \forall n \in \mathbb{N}$ ). $f$ is weakly mixing, so there exists $k \in \mathbb{N}$ such that

$$
(f \times f \times f \times f)^{k}(U \times U \times V \times V) \cap U \times V \times U \times V \neq \varnothing .
$$


We obtain that

$$
\begin{aligned}
& f^{k}(U) \cap U \neq \varnothing \quad \text { and } \quad f^{k}(U) \cap V \neq \varnothing \text {, } \\
& f^{k}(V) \cap U \neq \varnothing \quad \text { and } \quad f^{k}(V) \cap V \neq \varnothing .
\end{aligned}
$$

Therefore, $f^{k}(U)$ is a connected set that intersects the two connected sets $U$ and $V$. Thus, it contains the point $x \in D$ which satisfies that $U \cup V \cup\{x\}$ is a connected set or the minimal connected arc of $U$ and $V$. It is the same situation for $f^{k}(V)$. We thus have $f^{k}(U) \cap f^{k}(V) \neq \varnothing$. Hence, $f$ is weakly blending.

Remark 2. On dendrites, weakly mixing does not imply strongly blending. To illustrate this, we use (Example 17) in [22], which is a weakly mixing but not mixing dendrite map. Without loss of generality, let $U$ and $V$ be two open, connected and disjoint sets. According to the definition of the map, $\forall m \in \mathbb{N}, f^{m}(U) \cap f^{m}(V)=\varnothing$ or $\{o\}$, where o is the only periodic point of the map. Therefore, the map is not strongly blending.

Now, we show that weakly mixing does not only imply weakly blending but also strongly blending whenever $D$ is a dendrite whose branch points are not dense.

Theorem 11. On dendrites whose sets of branch points is not dense, weakly mixing implies strongly blending.

Proof. Without loss of generality, assume that $U$ and $V$ are two non-empty connected open sets in $D$ whose branch points are not dense. Let $E$ be a disconnecting interval in $D$ and $E_{1}, E_{2}$ and $E_{3}$ are three disjoint open sets in $E$, where $E_{2}$ lies between $E_{1}$ and $E_{3}$. Since $f$ is weakly mixing, there exists $m \in \mathbb{N}$ such that

$$
(f \times f \times f \times f)^{m}(U \times U \times V \times V) \cap E_{1} \times E_{3} \times E_{1} \times E_{3} \neq \varnothing .
$$

This implies that

$$
f^{m}(U) \cap E_{i} \neq \varnothing \quad \text { and } \quad f^{m}(V) \cap E_{i} \neq \varnothing \quad \text { for } i=1,3 .
$$

Now, $f^{m}(U)$ is a connected set that intersects the two connected sets $E_{1}$ and $E_{3}$ so it contains $E_{2}$. It is the same case for $f^{m}(V)$. Therefore, $f^{m}(U) \cap f^{m}(V) \supseteq E_{2}$, which is an open set. Thus, $f$ is strongly blending.

Theorem 12. Let $f$ be an interval map. Then, $f$ is weakly mixing if and only if $f$ is strongly blending and transitive.

Proof. From Theorems 7 and 11.

Finally, some results relate to Devaney chaos.

Theorem $13([28])$. Let $(X, f)$ be a non-trivial dynamical system. Then, weakly mixing implies SDIC.

Proposition 2. On dendrites, Auslander and Yorke chaos does not imply Devaney chaos.

Proof. In [22], Hohen and Mouron constructed a weakly mixing dendrite map that contains only one periodic point [23]. This map is chaotic in the sense of Auslander and Yorke, since it is transitive and has SDIC by Theorem 13. Therefore, Auslander Yorke chaos does not imply Devaney chaos.

Theorem 14 ([8]). Let $f$ be a map on a compact metric space with no isolated points. Then, Devaney chaos implies strong dense periodicity. 
Theorem 15. On dendrites, Devaney chaos implies strong dense periodicity.

Proof. Since $D$ is infinite, compact and $f$ is transitive, the space $D$ has no isolated points. By Theorem 14 , $f$ has a strong dense periodicity.

Proposition 3. On dendrites, strong dense periodicity does not imply Devaney chaos.

Proof. Let $f(x)=-h(x)$, which was represented in Example 1. $\left.f\right|_{[0,1]}$ and $\left.f\right|_{[-1,0]}$ are Devaney chaotic, so $P_{m}\left(\left.f\right|_{[0,1]}\right)$ and $P_{m}\left(\left.f\right|_{[-1,0]}\right)$ are dense $\forall m \in \mathbb{N}$ on $[0,1]$ and $[-1,0]$, respectively. Hence,

$$
P_{m}(f)=P_{m}\left(\left.f\right|_{[0,1]}\right) \cup P_{m}\left(\left.f\right|_{[-1,0]}\right)
$$

is dense $\forall m \in \mathbb{N}$ on $[-1,1]$. It was proven that $f$ is not transitive, since $f([0,1])=[0,1])$, which means that $[0,1]$ is invariant under $f[7]$. Therefore, $f$ is not Devaney chaotic.

\section{Conclusions}

We know that, on interval maps, mixing coincides with locally everywhere onto if and only if the endpoints of the interval are accessible. In our paper, we discussed this relation on dendrites. We proved that locally everywhere onto implies mixing and accessibility of endpoints for dendrites that do not have a dense set of branch points. A counterexample was given to show that this result does not hold for every dendrite. The converse was proven on trees, intervals and also on dendrites with a taxicab metric.

For compact space, we proved that any map has a transitive point whose every open set containing it covers all the space after some iteration is locally everywhere onto. Moreover, we showed that a strongly blending transitive compact map implies weakly mixing.

On dendrites, we investigated that weakly mixing implies weakly blending and strongly blending on dendrites whose branch points are not dense. In addition, we concluded that Devaney chaos implies strong dense periodicity while the converse is not true, which is the same as intervals. However, unlike intervals, Auslander and Yorke chaos does not imply Devaney chaos on dendrites.

Author Contributions: A.F. and S.C.D.-K. cooperated on completing this research.

Funding: This research was funded by a grant from Universiti Kebangsaan Malaysia.

Acknowledgments: We are indebted to Universiti Kebangsaan Malaysia for providing financial support and facilities for this research under the grants FRGS/1/2017/STG06/UKM/02/2 and GUP-2019-054

Conflicts of Interest: The authors declare no conflict of interest.

\section{References}

1. Devaney, R.L. An Introduction to Chaotic Dynamical Systems, 2nd ed.; Addison-Wesley Publishing Company: Redwood City, CA, USA, 1989.

2. Banks, J.; Brooks, J.; Cairns, G.; Davis, G.; Stacey, P. On Devaney's definition of chaos. Am. Math. Mon. 1992, 99, 332-334. [CrossRef]

3. Vellekoop, M.; Berglund, R. On intervals, transitivity = chaos. Am. Math. Mon. 1994, 101, 353-355. [CrossRef]

4. Auslander, J.; Yorke, J.A. Interval maps, factors of maps, and chaos. Tohoku Math. J. 1980, 32, 177-188. [CrossRef]

5. Crannell, A. The role of transitivity in Devaney's definition of chaos. Am. Math. Mon. 1995, 102, 788-793. [CrossRef]

6. Baloush, M. On Some Chaos Notions of Dynamical Systems. Ph.D. Thesis, Universiti Kebangssan Malaysia, Bangi, Malaysia, 2019.

7. Dzul-Kifli, S.C. Chaotic Dynamical Systems. Ph.D. Thesis, University of Birmingham, Birmingham, UK, 2011. 
8. Dzul-Kifli, S.C.; Good, C. On Devaney chaos and dense periodic points: period 3 and higher implies chaos. Am. Math. Mon. 2015, 122, 773-780. [CrossRef]

9. Dzul-Kifli, S.C. and Good, C. The chaotic behavior on the unit circle. Int. J. Math. Anal. 2016, 10, 1245-1254. [CrossRef]

10. Baloush, M.; Dzul-Kifli, S.C. On some strong chaotic properties of dynamical system. In Proceeding of the 4th International Conference on Mathematical Science, Selangor, Malaysia, 15-17 November 2016.

11. Baloush, M.; Dzul-Kifli, S.C. Implication diagram of five chaos characterizations: a survey on compact metric space and shift of finite type. JQMA 2017, 13, 15--23.

12. Banks, J. Regular periodic decompositions for topologically transitive maps. Ergod. Theor. Dyn. Syst. 1997, 17, 505-529. [CrossRef]

13. Banks, J.; Trotta, B. Weak mixing implies mixing for maps on topological graphs. J. Differ. Equ. Appl. 2005, 11, 1071-1080. [CrossRef]

14. Nadler, S. Continuum Theory: An Introduction; Marcel Dekker, Inc.: New York, NY, USA, 1992.

15. Charatonik, J. J.; Charatonik, W. J. Dendrites. Aportaciones Mat. Comun. 1998, 22, 227-253.

16. Alseda, L.; Kolyada, S.; Llibre, J.; Snoha, L. Entropy and periodic points for transitive maps. Trans. Am. Math. Soc. 1999, 351, 1551-1573. [CrossRef]

17. Brian, W.; Meddaugh, J.; Raines, B. Shadowing is generic on dendrites. Discret. Contin. Dyn. 2019. [CrossRef]

18. Roth, Z. Distributional Chaos and Dendrites. Int. J. Bifurcat. Chaos 2018, 28. [CrossRef]

19. Abouda, H.; Naghmouchi, I. 2017. A note on periodic points of dendrite maps and their induced maps. J. Math. Anal. Appl. 2017 448, 722-725. [CrossRef]

20. Mai, J.; Shi, E. $\bar{P}=\bar{R}$ for maps of dendrites $X$ with $\operatorname{Card}(\operatorname{End}(X))<$ c. Int. J. Bifurcat. Chaos 2009, 19, 1391-1396.

21. Dirbák, M.; Snoha, L'.; Ŝpitalsky, V. Minimalityý, transitivity, mixing and topological entropy on spaces with a free interval. Ergod. Theor. Dyn. Syst. 2013, 33, 1786-1812. [CrossRef]

22. Hoehn, L.; Mouron, C. Hierarchies of chaotic maps on continua. Ergod. Theor. Dyn. Syst. 2014, 34, 1897-1913. [CrossRef]

23. Acosta, G.; Hernández-Gutiérrez, R.; Naghmouchi, I.; Oprocha, P. Periodic points and transitivity on dendrites. Ergod. Theor. Dyn. Syst. 2017, 37, 2017-2033. [CrossRef]

24. Blokh, A. M. (1995). The "Spectral" Decomposition for One-Dimensional Maps; Dynamics Reported; Springer: Berlin/Heidelberg, Germany, 1995; pp. 1-59.

25. Ruette, S. Chaos on the Interval, a Survey of Relationship between the Various Kinds of Chaos for Continuous Interval Maps. Avalable online: https://www.math.u-psud.fr/ ruette/articles/chaos-int.pdf (accessed on 18 January 2019).

26. Banks, J. Chaos for induced hyperspace maps. Chaos Soliton Fract. 2005, 25, 681-685. [CrossRef]

27. Furstenberg, H. Disjointness in ergodic theory, minimal sets, and a problem in Diophantine approximation. Theor. Comput. Syst. 1967, 1, 1-49. [CrossRef]

28. Akin, E.; Kolyada, S. Li-Yorke sensitivity. Nonlinearity 2003, 16, 1421-1433. [CrossRef]

(C) 2019 by the authors. Licensee MDPI, Basel, Switzerland. This article is an open access article distributed under the terms and conditions of the Creative Commons Attribution (CC BY) license (http:/ / creativecommons.org/licenses/by/4.0/). 